\title{
REACCIÓN DE SIETE CULTIVARES DE CAPSICUM A DIFERENTES DENSIDADES DEL NEMATODO DEL NÓDULO, Meloidogyne incógnita (KOFOID \& WHITE 1919) CHITWOOD 1949
}

\author{
REACTION TO SEVEN CAPSICUM CULTIVARS OF DIFFERENT DENSITIES NODULE \\ NEMATODE, Meloidogyne incógnita (KOFOID \& WHITE 1919) 1949 CHITWOOD
}

${ }^{1}$ Fátima M. García Rodríguez y ${ }^{2}$ Angel Alfonso Palomo Herrera

\begin{abstract}
Resumen
Se evaluaron los cultivares Chinto, Fresnillo y Mitla (Capsicumannuum); Charapita, Limo y Panca (Capsicumchinense) y Escabeche (Capsicumbaccatumvar.pendullum),para determinar su reacción frente a diferentes densidades poblacionales del nematodo Meloidogyne incógnita a nivel de invernadero. Teniendo en cuenta la escala de nodulación del Proyecto Internacional Meloidogyne, se determino que los cultivares Mitla, Fresnillo y Panca presentaron mayor tolerancia al ataque del nematodo, presentado un índice de nodulación promedio de 4.38, 4.67 y 4.00, respectivamente. Los cultivares Chinto y Limo presentaron una tolerancia moderada con un índice de nodulación de 4.90, mientras que los cultivares Charapita y Amarillo presentaron el menor grado de tolerancia al daño de altas densidades de $M$. incognita, siendo observado un índice de nodulación de 4.96 y 4.93, respectivamente,comportándose como susceptibles.
\end{abstract}

Palabras clave: Meloidogyne incognita, Resistencia, Nematodo.

\begin{abstract}
CultivarsChinto, Fresnillo and Mitla (Capsicumannuum); Charapita, Limo and Panca (Capsicumchinense) and Yellowor Escabeche (Capsicumbaccatumvar. pendullum) wereevaluatedto determine theirreaction to different populationdensities of thenemato de Meloidogyne incognita at greenho use conditions. Consideringthenodulationscale of the International Meloidogyne Project, itwasdeterminedthatcultivars Mitla, Fresnillo and Panca showedhighertolerance of nematodeattack, presented a nodulationindexaverage 4.38, 4.67 and 4.0, respectively Thecultivars Chinto and Limo showed a moderatetolerance of nodulationindex of 4.90, while Charapita and Amarillo cultivars showedt helowestdegree of damagetolerance of highdensities of M. incognita, withanindexnodulation of 4.96 and 4.93, respectively, with a behavoir as susceptible.
\end{abstract}

Key Words: Meloidogyne incognita, Capsicum, Resistance, Nematode.

\section{Introducción}

El género Capsicum a grupa a hortalizas de importancia a nivel mundial, siendo incluidas especies con los nombres de ají, chile, rocoto, pimiento, entre otros; este género oriundo de América, se caracteriza por su sabor, no siempre picante; siendo en el Perúrepresentado por las especies Capsicumannum, Capsicumpubescens, Capsicumfrutescens, Capsicumbaccatum $\mathrm{y}$ Capsicumchinense $(3,4,12,14)$.

Estos cultivos son afectados por plagas y enfermedades, siendoel nematodo del nódulo de la raíz, Meloidogyne incognita (Kofoid\& White), uno de los mayores limitantes en la producción mundial afectando la calidad y el rendimiento de estas especies vegetales $(16,19,20)$.

${ }^{1}$ Ingeniero Agrónomo - UNALM

${ }^{2}$ Departamento Fitopatología, Facultad Agronomía - UNALM, Lima, Perú. E-mail: fonz@lamolina.edu.pe
Entre las principales medidas de control para nematodos, una de las medidas más recomendadas y eficientes es el uso de variedades resistentes $(2,10,15)$. La presente investigación se realiza con la finalidad de identificar el comportamiento de siete especies de Capsicum bajo diferentes densidades poblacionales de Meloidogyne incognita (Kofoid\& White) bajo condiciones de invernadero.

\section{Materiales y Métodos}

El estudio se realizó en el Invernadero de Nematología y en el Laboratorio de Nematología "M. Oostenbrink" de la Universidad Nacional Agraria La Molina. 
Material Vegetal: se utilizaron 7 cultivares del genero Capsicum.

- Chinto,FresnilloyMitlacultivares de Capsicumannuum,

- Charapita, Limo y Panca cultivares de Capsicumchinense

- Escabechecultivares de Capsicumbaccatumvar. pendullum.

Todas las semillas fueron colectadas localmente con agricultores en Huaral, Cañete, Moyobamba y tiendas agrícolas comerciales.

\section{Metodología}

Las semillas fueron llevadas a un vivero comercial para su propagación y después de 40 días se trasplantaron a bolsas de polietileno, conteniendo $950 \mathrm{cc}$. de sustrato pausterizado obtenido de la mezcla de arena y suelo de chacra (1:1). Antes de realizar el trasplante el suelo fue humedecido para evitar que las plantas se estres en.

El inoculo de $M$. incógnita, fue recolectado a partir de raíces noduladasde tomate del cv. Rió Grande. La extracción de huevos se realizó según el método propuesto por Hussey y Barker (1973).Dos semanas después del trasplante se realizó la inoculación de las plantas con las densidades poblacionales de 0 (testigo), $10,100,250,500,1000,2000$ huevos/100 cc. de suelo y se completaron las bolsas a $1000 \mathrm{cc}$. de sustrato.

La evaluación se realizó a los 79 días después de realizada la inoculación. Los parámetros evaluados fueron:

Parámetros Indirectos de Crecimiento de la Planta: altura de planta $(\mathrm{cm})$; peso fresco de la parte aérea (g.); peso seco de la parte aérea (g.); peso fresco de raíz (g.); biomasa total (g.); volumen radicular (ml.).

Parámetros Indirectos de Respuesta del Hospedante al Nematodo: Las raíces fueron evaluadas, mediante 3 escalas de nodulación:

- Escala de Barker, según la cual se asignan valores de 1 a 10, donde:

Tabla 1. Escala de Nodulación de Barker.

\begin{tabular}{lc}
\hline $\begin{array}{c}\text { Grado de } \\
\text { Nodulación }\end{array}$ & \% de Nódulos en el Sistema radicular \\
\hline 0 & Raíces totalmente sanas \\
1 & $10 \%$ \\
2 & $20 \%$ \\
3 & $30 \%$ \\
4 & $40 \%$ \\
5 & $50 \%$ \\
6 & $60 \%$ \\
7 & $70 \%$ \\
8 & $80 \%$ \\
9 & $90 \%$ \\
10 & $100 \%$ \\
\hline
\end{tabular}

- Escala del Proyecto Internacional Meloidogyne (PIM), donde:
Tabla2. Escala de Nodulación del PIM.

\begin{tabular}{cl}
\hline $\begin{array}{c}\text { Grado de } \\
\text { Nodulación }\end{array}$ & \multicolumn{1}{c}{ No de Nódulos o Masas de Huevos $^{\circ}$} \\
\hline 0 & Sin nódulos o Masas de huevos \\
1 & $1-2$ Nódulos \\
2 & $3-10$ Nódulos \\
3 & $11-30$ Nódulos \\
4 & $31-100$ Nódulos \\
5 & +100 Nódulos o masas de huevos \\
\hline
\end{tabular}

- $\quad$ Escala de Zeck, en el cual asigna valores de 0 a 10, de acuerdo a la siguiente Tabla

Tabla 3. Escala de Nodulación de Zeck.

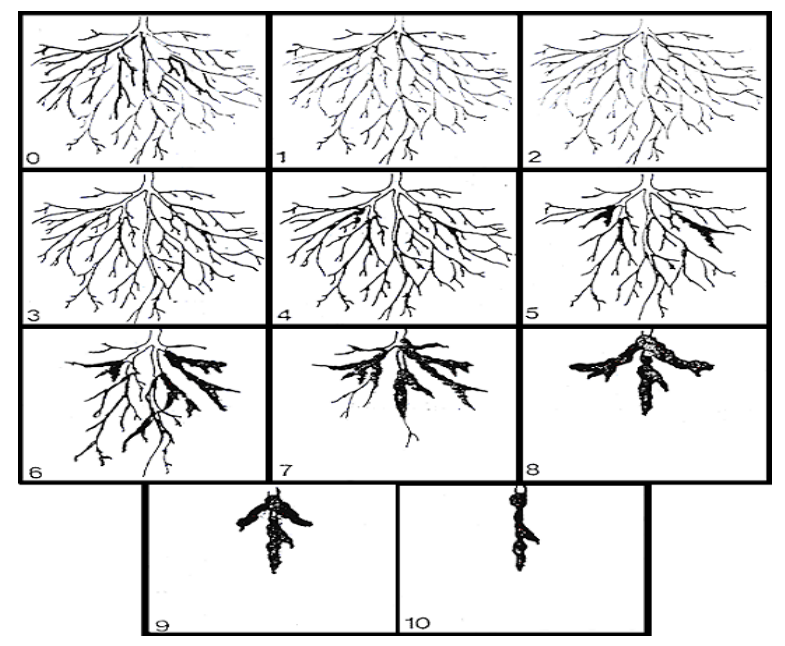

Donde:

$0=$ Sistema radicular completamente sano, no infestado.

1 = Muy pocos nódulos pequeños pueden detectarse.

2 = Nódulos pequeños como en 1 , en mayor numero y fáciles de detectar.

3 = Numerosos nódulos pequeños, algunos crecimientos juntos, no afectara seriamente a la fusión de las raíces.

4=Numerosos nódulos pequeños o algunos grandes, la gran mayoría de raíces funcionales.

$5=25 \%$ del sistema radicular seriamente nodulados y no funcional.

$6=50 \%$ del sistema radicular seriamente nodulados y no funcional.

$7=75 \%$ del sistema radicular seriamente nodulados y no funcional.

$8=$ No hay raíces sanas, alimentación de planta interrumpida, planta aun verde.

$9=$ Sistema radicular completamente nodulado y podrido, planta moribunda.

$10=$ Planta y raíces muertas 
Parámetros Directos de Reproducción del Nematodo

Número de huevos por gramos de raíz: La obtención de huevos se realizó usando el método del hipoclorito de sodio a una concentración del $0.5 \%$ obteniéndose el número total de huevos por raíz.

Numero de juveniles 2 (J2) en 100 cc de suelo: La obtención de los J2 a partir del sustrato, se realizó mediante el método de centrifugación en azúcar para 250 cc.de suelo.

Población Final: Con la información del número de huevos en raíz y el número de $\mathrm{J} 2$ por sustrato se obtiene la población final de nematodos.

Tasa de Reproducción (Pf/Pi): Este parámetro resulta de dividir la población final de nematodos y la población inicial inoculada.

Determinación de la Eficiencia del Hospedante en la Reproducción del Nematodo: De acuerdo al sistema de evaluación propuesto por V. Dropkin modificado por Canto Sáenz, donde se toma en cuenta que la reproducción del nematodo (tasa de reproducción PF/Pi) y el análisis estadístico del daño producido por el nematodo en la planta.
El diseño experimental utilizado fue un Diseño Completo al Azar (DCA), con siete tratamientos (7densidades poblacionales) y 5 repeticiones por cada variedad, siendo la unidad experimental una planta. Las comparaciones de medias se hicieron mediante la prueba de Duncan. Los datos obtenidos durante el experimento fueron transformados según las recomendaciones hechas por Noé (1985), para experimentos nematológicosutilizando

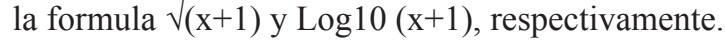

\section{Resultados}

En las Tablas 7, 8 y 9; y los gráficos 1, 2, 3, 4 y 5 se observan los resultados obtenidos en el estudio para cada uno de los parámetros evaluados.

La reacción de cultivares de Capsicumsp. aMeloidogyne incognita según el Sistema de Evaluación propuesto por V. Dropkin modificado por Canto-Sáenz se observan en las tablas 4,5 y 6 .

Tabla 4. Escala para Evaluar la Reacción de la Planta al género Meloidogyne, propuesta por V. Dropkin y modificada por Canto-Sáenz según el Peso Seco del Follaje.

\begin{tabular}{lll}
\hline Reproducción del Nematodo & \multicolumn{2}{c}{ Daño del Nematodo en la Planta } \\
\cline { 2 - 3 } & Estadísticamente significativa & Estadísticamente no significativo \\
\hline Hospedante Eficiente & SUSCEPTIBLE $/$ Pi $>1.5)$ & TOLERANTE \\
& AMARILLO & CHARAPITA, \\
& & CHINTO, \\
& & FRESNILLO, \\
Hospedante no Eficiente & LIMO, MITLA \\
$(\mathrm{Pf} / \mathrm{PI}<1.5)$ & PANCA \\
\hline
\end{tabular}

Tabla 5. Escala para Evaluar la Reacción de la Planta al género Meloidogyne, propuesta por V. Dropkin y modificada por Canto-Sáenz según el Peso Radicular (peso fresco).

\begin{tabular}{lll}
\hline Reproducción del Nematodo & \multicolumn{2}{c}{ Daño del Nematodo en la Planta } \\
\cline { 2 - 3 } $\begin{array}{l}\text { Hospedante Eficiente } \\
(\mathrm{Pf} / \mathrm{Pi}>1.5)\end{array}$ & Estadísticamente significativa & Estadísticamente no significativo \\
\cline { 2 - 3 } & CUSCEPTIBLE & TOLERANTE \\
& & AMARILLO, \\
& & CHINTO, \\
& & FRESNILLO, \\
Hospedante no Eficiente & LIMO, MITLA, \\
$(\mathrm{Pf} / \mathrm{PI}<1.5)$ & HIPERSUSCEPTIBLE & PANCA \\
\hline
\end{tabular}


Tabla 6. Escala para Evaluar la Reacción de la Planta al género Meloidogyne, propuesta por V. Dropkin y modificada por Canto-Sáenz según la Biomasa Total (peso fresco).

\begin{tabular}{lll}
\hline Reproducción del Nematodo & \multicolumn{2}{c}{ Daño del Nematodo en la Planta } \\
\cline { 2 - 3 } & Estadísticamente significativa & Estadísticamente no significativo \\
\hline Hospedante Eficiente & SUSCEPTIBLE $/ \mathrm{Pi}>1.5)$ & TOLERANTE \\
& & CHINTO, \\
& & CHARAPITA. \\
& & FRESNILLO. \\
Hospedante no Eficiente & LIMO, MITLA, \\
$(\mathrm{Pf} / \mathrm{PI}<1.5)$ & HIPERSUSCEPTIBLE & RANCA \\
\hline
\end{tabular}

\section{Discusión}

La resistencia divide a las plantas en categorías, desde cultivares altamente resistentes, aquellos que limitan el desarrollo y reproducción del nematodo; cultivares susceptibles que permiten el desarrollo y reproducción del nematodo libremente, viendo perjudicado el crecimiento de la planta; y cultivares tolerantes donde el nematodo puede desarrollarse sin problemas sin producirse una reducción del rendimiento de la planta o la calidad de los frutos. El grado de resistencia es un indicativo de la habilidad del nematodo para reproducirse sobre una planta hospedante.

Tabla 7. Parámetros Indirectos del Creciemiento de cultivares de Capsicum después de inoculadas con M. incognita.

\begin{tabular}{|c|c|c|c|c|c|c|c|c|}
\hline Parámetros Indirectos & $\begin{array}{l}\text { Ind./100 cc. } \\
\text { de suelo }\end{array}$ & Chinto & Fresnillo & Mitla & Charapita & Limo & Panca & Amarillo \\
\hline \multirow{8}{*}{ Peso Fresco de Follaje (g.) } & 0 & 9.4 & 22.4 & 30.3 & 12.2 & $7.5 \mathrm{c}$ & 22 & $41.7 \mathrm{ab}$ \\
\hline & 10 & 11.2 & 11.7 & 27.3 & 12 & $8.9 \mathrm{abc}$ & 18.7 & $30.4 \mathrm{bc}$ \\
\hline & 100 & 8.5 & 25.1 & 30.5 & 14.1 & $12.48 \mathrm{ab}$ & 17.9 & $36.16 \mathrm{abc}$ \\
\hline & 250 & 15.7 & 21.3 & 29.1 & 16.6 & $12.36 \mathrm{ab}$ & 18.7 & $34.4 \mathrm{bc}$ \\
\hline & 500 & 10 & 22.2 & 29 & 11.5 & $8.94 \mathrm{bc}$ & 18.4 & $27 \mathrm{c}$ \\
\hline & 1000 & 12.1 & 21.3 & 32.4 & 10.9 & $14.18 \mathrm{a}$ & 24.4 & $34.78 \mathrm{bc}$ \\
\hline & 2000 & 10.4 & 24.7 & 32.1 & 17.6 & $10.98 \mathrm{abc}$ & 23.5 & $47.16 \mathrm{a}$ \\
\hline & C.V \% & 16.2 & 13.5 & 8.8 & 18.1 & 12.5 & 12.4 & 10.8 \\
\hline \multirow{8}{*}{ Peso Seco de Follaje (g.) } & 0 & 2.3 & 4.6 & 6 & 2.2 & 2 & 4.6 & $6.28 \mathrm{abc}$ \\
\hline & 10 & 2.9 & 3.1 & 6.7 & 1.1 & 2.5 & 3.4 & $5.4 \mathrm{bc}$ \\
\hline & 100 & 1.9 & 5 & 6.5 & 2.4 & 3.1 & 4.1 & $7.32 \mathrm{ab}$ \\
\hline & 250 & 3.8 & 4.2 & 5.8 & 2.9 & 2.6 & 3.7 & $5.78 \mathrm{bc}$ \\
\hline & 500 & 2.1 & 4 & 5.2 & 1.9 & 2 & 3.7 & $4.98 \mathrm{c}$ \\
\hline & 1000 & 2.5 & 3.9 & 6.1 & 1.9 & 2.7 & 4.8 & $6.06 \mathrm{bc}$ \\
\hline & 2000 & 2.1 & 4.7 & 6 & 2.7 & 2.4 & 4.8 & $7.98 \mathrm{a}$ \\
\hline & C.V \% & 14 & 10.5 & 5.9 & 18.2 & 10.6 & 12.6 & 8.7 \\
\hline \multirow{8}{*}{ Peso Fresco de Raíz (g.) } & 0 & 12.5 & 8.9 & 20.5 & $22.66 \mathrm{a}$ & 12.3 & 19.2 & 22.5 \\
\hline & 10 & 14.4 & 13.9 & 21.5 & $18.6 \mathrm{abc}$ & 13.4 & 29.2 & 25.4 \\
\hline & 100 & 12.9 & 10 & 19.9 & $14.22 \mathrm{bc}$ & 11.8 & 18.2 & 21 \\
\hline & 250 & 15.5 & 10.5 & 16.3 & $16.6 \mathrm{abc}$ & 14.4 & 21.3 & 15.1 \\
\hline & 500 & 13.1 & 8.5 & 14.3 & $10.84 \mathrm{c}$ & 11.6 & 16.6 & 14.6 \\
\hline & 1000 & 10.9 & 7.3 & 12.9 & $11.82 \mathrm{ab}$ & 13.4 & 19.7 & 18.5 \\
\hline & 2000 & 15.8 & 11.5 & 13.8 & $20.46 \mathrm{ab}$ & 14.8 & 21.8 & 23.7 \\
\hline & C.V \% & 9.5 & 17.1 & 15.6 & 18.6 & 14.7 & 18.3 & 19.4 \\
\hline \multirow{8}{*}{ Biomasa Total (g.) } & 0 & 21.8 & 31.3 & 50.8 & 34.8 & 19.8 & 41.2 & $64.16 \mathrm{ab}$ \\
\hline & 10 & 25.2 & 32.9 & 52.2 & 33.8 & 23.5 & 50.7 & $56.74 \mathrm{abc}$ \\
\hline & 100 & 21.4 & 35.1 & 50.4 & 28.3 & 24.3 & 36.1 & $57.16 \mathrm{abc}$ \\
\hline & 250 & 31.2 & 31.8 & 45.4 & 33.2 & 26.7 & 40.1 & $49.46 \mathrm{bc}$ \\
\hline & 500 & 23.1 & 30.8 & 43.3 & 22.4 & 20.6 & 35.1 & $41.64 \mathrm{c}$ \\
\hline & 1000 & 23 & 28.6 & 45.3 & 22.7 & 27.6 & 44.1 & $53.24 \mathrm{abc}$ \\
\hline & 2000 & 26.3 & 36.2 & 45.9 & 38.1 & 25.8 & 45.3 & $70.86 \mathrm{a}$ \\
\hline & C.V \% & 11.5 & 11.8 & 8.4 & 17.4 & 12.6 & 12.9 & 11.4 \\
\hline
\end{tabular}


REACCIÓNREACCIÓN DE SIETE CULTIVARES DE CAPSICUM A DIFERENTES DENSIDADES DEL NEMATODO DEL NÓDULO, Meloidogyne incógnita (KOFOID \& WHITE 1919) CHITWOOD 1949

Julio-Diciembre 2016

Tabla 8. Grado de Nodulación en cultivares de Capsicum después de inoculados con M.

\begin{tabular}{|c|c|c|c|c|c|c|c|c|}
\hline $\begin{array}{l}\text { Escalas de } \\
\text { Nodulación }\end{array}$ & $\begin{array}{l}\text { Ind./100 cc de } \\
\text { suelo }\end{array}$ & Chinto & Fresnillo & Mitla & Charapita & Limo & Panca & Amarillo \\
\hline & 10 & 4.6 & $3 \mathrm{~b}$ & $3 \mathrm{c}$ & 4.8 & 4.6 & $3.6 \mathrm{~b}$ & $4.6 \mathrm{~b}$ \\
\hline & 100 & 4.8 & 05:00 a.m. & $4 \mathrm{~b}$ & 5 & 4.8 & $4.8 \mathrm{a}$ & 05:00 a.m. \\
\hline Proyecto & 250 & 5 & 05:00 a.m. & $4 \mathrm{~b}$ & 5 & 5 & $4.8 \mathrm{a}$ & 05:00 a.m. \\
\hline Internacional & 500 & 5 & 05:00 a.m. & 05:00 a.m. & 5 & 5 & 05:00 a.m. & 05:00 a.m. \\
\hline \multirow[t]{3}{*}{ Meloidogyne } & 1000 & 5 & 05:00 a.m. & 05:00 a.m. & 5 & 5 & 05:00 a.m. & 05:00 a.m. \\
\hline & 2000 & 5 & 05:00 a.m. & 05:00 a.m. & 5 & 5 & 05:00 a.m. & 05:00 a.m. \\
\hline & C.V. & 5.89 & 0 & 0 & 3.68 & 5.89 & 7.27 & 4.53 \\
\hline $\begin{array}{l}\text { Escalas de } \\
\text { Nodulación }\end{array}$ & $\begin{array}{c}\text { Ind./100 cc de } \\
\text { suelo }\end{array}$ & Chinto & Fresnillo & Mitla & Charapita & Limo & Panca & Amarillo \\
\hline \multirow{7}{*}{ Barker } & 10 & $1.8 \mathrm{~d}$ & $1.8 \mathrm{~d}$ & $1 \mathrm{~d}$ & $1.8 \mathrm{e}$ & $1.6 \mathrm{~d}$ & $1 \mathrm{~d}$ & $1 \mathrm{~d}$ \\
\hline & 100 & $3.6 \mathrm{c}$ & $4.2 \mathrm{c}$ & $2.8 \mathrm{c}$ & $3.4 \mathrm{~d}$ & $3.8 \mathrm{c}$ & $3.4 \mathrm{bc}$ & $3.8 \mathrm{c}$ \\
\hline & 250 & $4.2 \mathrm{bc}$ & $5.6 \mathrm{~b}$ & $4.6 \mathrm{~b}$ & $6.2 \mathrm{bc}$ & $5.6 \mathrm{ab}$ & $3 \mathrm{c}$ & $4.6 \mathrm{bc}$ \\
\hline & 500 & $5.2 \mathrm{a}$ & $8.8 \mathrm{a}$ & $5 \mathrm{ab}$ & 08:00 a.m. & $6.8 \mathrm{a}$ & $4.6 \mathrm{a}$ & 08:00 a.m. \\
\hline & 1000 & $5.2 \mathrm{~s}$ & $6 \mathrm{~b}$ & $5.6 \mathrm{a}$ & $7 \mathrm{~b}$ & $6.4 \mathrm{a}$ & $4 \mathrm{ab}$ & $5.4 \mathrm{~b}$ \\
\hline & 2000 & $4.4 \mathrm{~b}$ & $5.2 \mathrm{~b}$ & $4.6 \mathrm{~b}$ & $5.4 \mathrm{c}$ & $5 \mathrm{bc}$ & $4 \mathrm{ab}$ & $5.2 \mathrm{~b}$ \\
\hline & C.V. & 13.84 & 11.23 & 13.13 & 12.18 & 19.31 & 17.75 & 15.15 \\
\hline \multirow{7}{*}{ Zeck } & 10 & $2.6 \mathrm{~b}$ & $3.2 \mathrm{~b}$ & $2 \mathrm{e}$ & $3.6 \mathrm{c}$ & $2.6 \mathrm{c}$ & $1.8 \mathrm{c}$ & $2.6 \mathrm{~d}$ \\
\hline & 100 & $3.2 \mathrm{~b}$ & $3.2 \mathrm{~b}$ & $3.2 \mathrm{~d}$ & $4.6 \mathrm{~b}$ & $3.8 \mathrm{~b}$ & $3.8 \mathrm{~b}$ & $4.6 \mathrm{c}$ \\
\hline & 250 & $5.6 \mathrm{a}$ & $3.2 \mathrm{a}$ & $6.2 \mathrm{a}$ & $5.8 \mathrm{a}$ & $5.8 \mathrm{a}$ & $3.8 \mathrm{~b}$ & $5 \mathrm{c}$ \\
\hline & 500 & 05:00 a.m. & $6.6 \mathrm{a}$ & $5.2 \mathrm{c}$ & $6.6 \mathrm{a}$ & $5.2 \mathrm{a}$ & $5.6 \mathrm{a}$ & 07:00 a.m. \\
\hline & 1000 & $5.6 \mathrm{a}$ & $6.2 \mathrm{a}$ & $5.8 \mathrm{ab}$ & $6.6 \mathrm{a}$ & $5.6 \mathrm{a}$ & 05:00 a.m. & $6.6 \mathrm{ab}$ \\
\hline & 2000 & $5.4 \mathrm{a}$ & 06:00 a.m. & $5.4 \mathrm{bc}$ & 06:00 a.m. & $5.6 \mathrm{a}$ & $5.6 \mathrm{a}$ & 06:00 a.m. \\
\hline & C.V. & 12.32 & 9.29 & 9.24 & 11.43 & 14.83 & 19.61 & 13.12 \\
\hline
\end{tabular}

Valores con la misma letra no presentan diferencia significativa

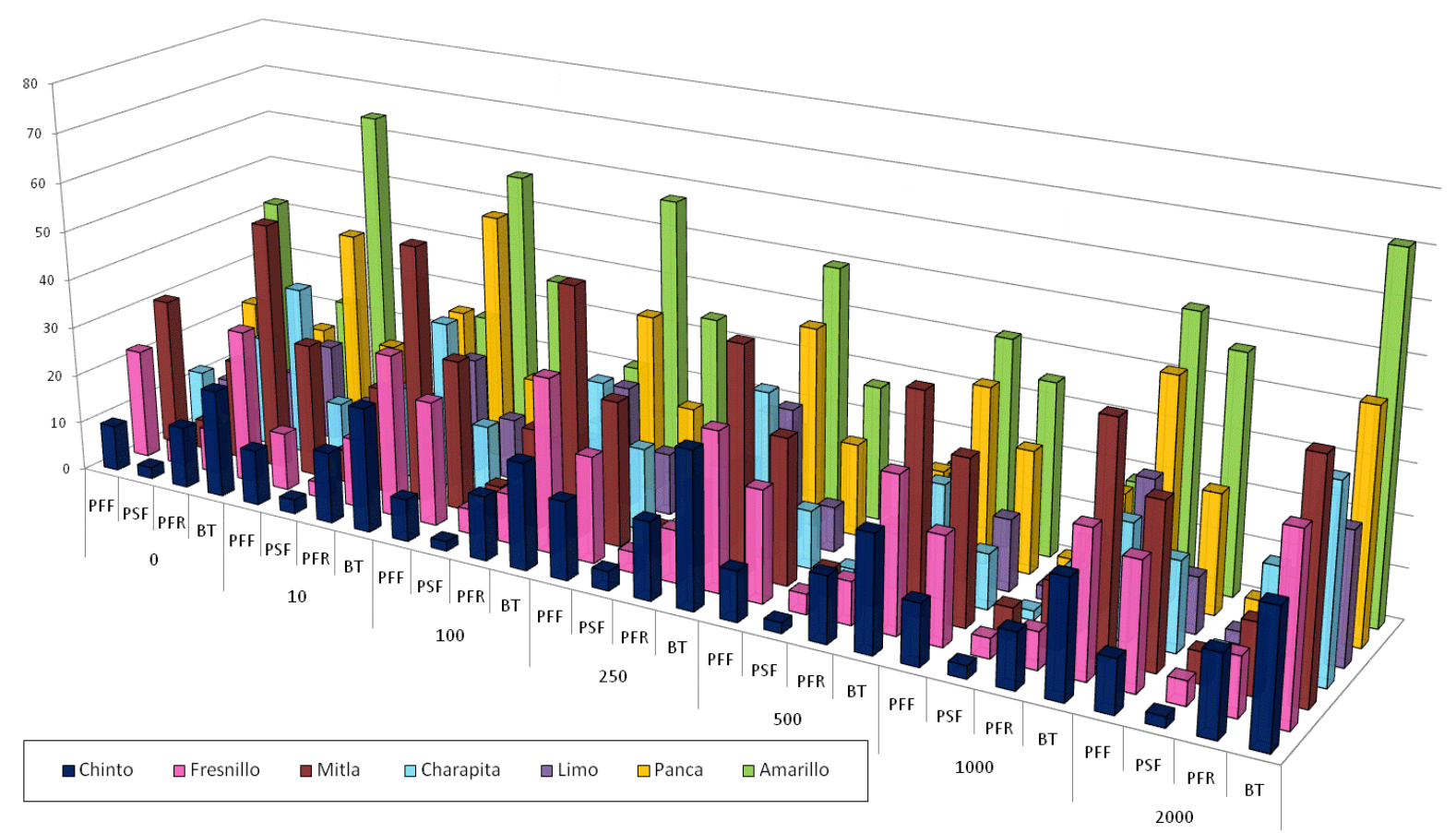

Figura 1. Parámetros Indirectos del Creciemiento de cultivares del genero CapsicumL. después de inoculadas con $\boldsymbol{M}$. incognita.

PFF: Peso Fresco del Follaje (g.) PSF: Peso Seco del Follaje (g.) PFR: Peso Fresco de Raiz (g.) BT: Biomasa Total (g.) 


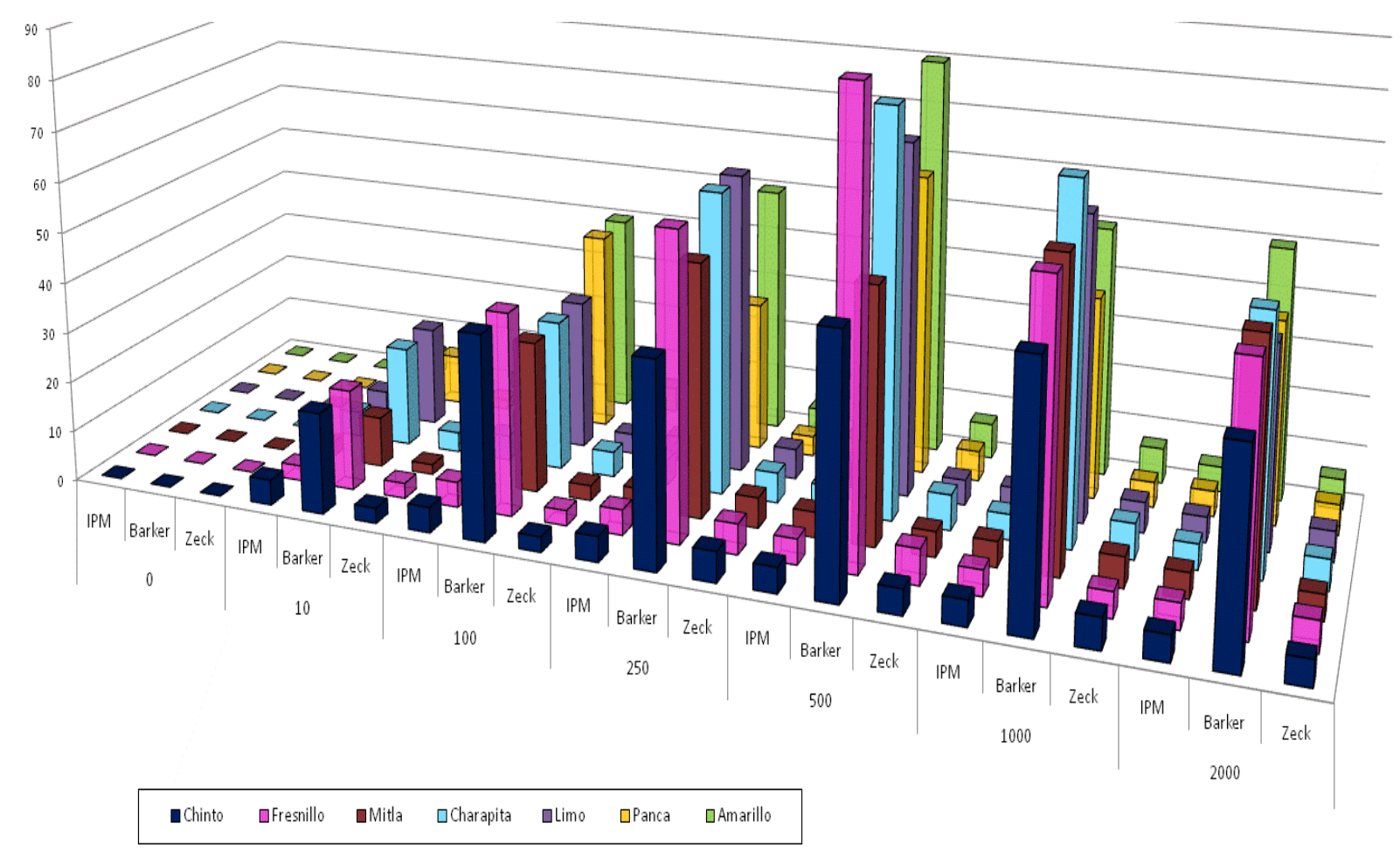

Figura 2. Grado de Nodulación de cultivares del genero Capsicumdespués de inoculadas con M. incognita.

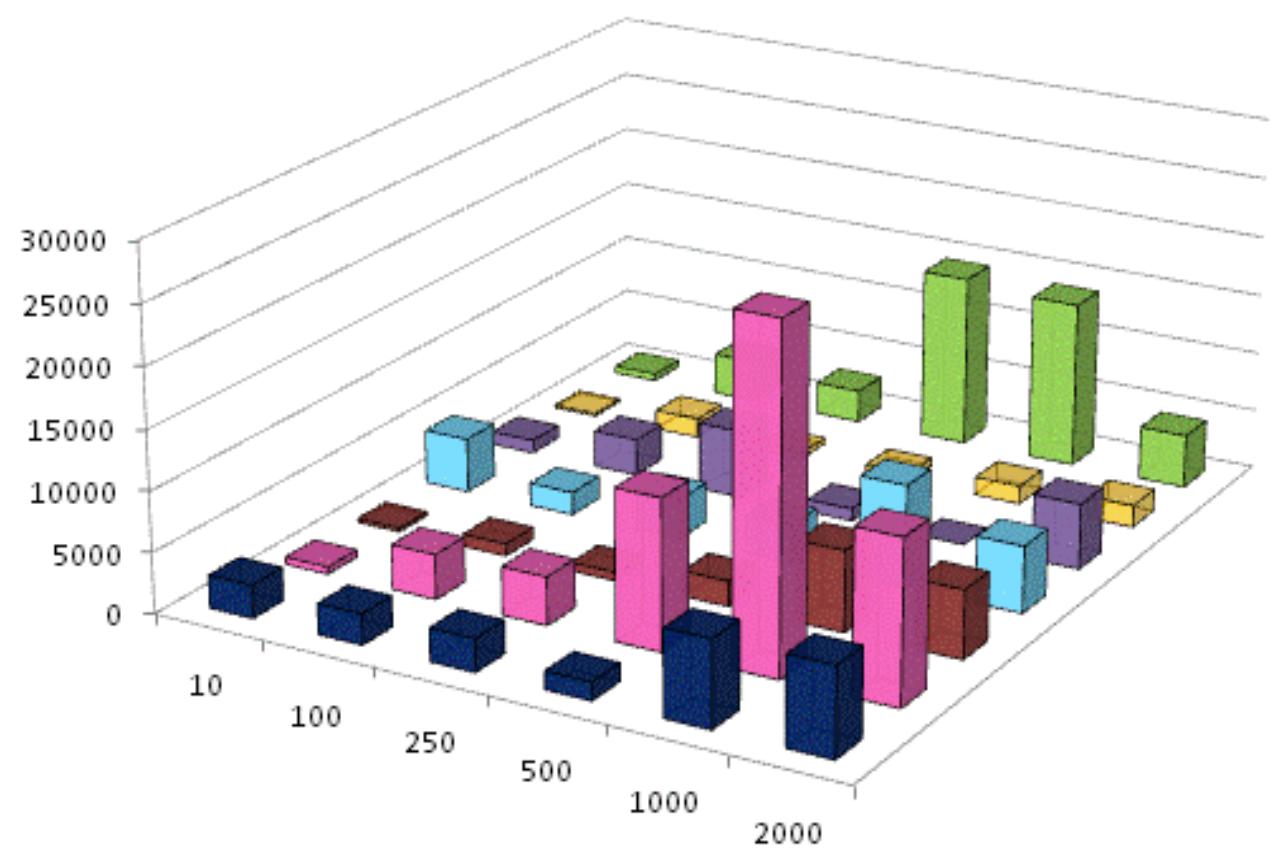

- Chinto afresnillo $\square$ Mitla $\square$ Charapita $\square$ Limo $\square$ Panca $\square$ Amarillo

Figura 3. Número de huevos por gramo de raíz en cultivares de Capsicum después de inoculados con diferentes densidades poblacionales de $M$. incognita. 
Tabla 9. Parámetros directos relacionados con la Reproduccion de $M$. incognita después de inoculados en cultivares de Capsicum.

\begin{tabular}{|c|c|c|c|c|c|c|c|c|}
\hline $\begin{array}{l}\text { Parámetros } \\
\text { Directos }\end{array}$ & $\begin{array}{c}\text { Ind./ } 100 \mathrm{cc} \\
\text { de suelo }\end{array}$ & Chinto & Fresnillo & Mitla & Charapita & Limo & Panca & Amarillo \\
\hline \multirow{7}{*}{$\begin{array}{c}\text { № gramos/ } \\
\text { gr. raíz }\end{array}$} & 10 & $2735.40 \mathrm{~b}$ & $594.80 \mathrm{c}$ & $309.60 \mathrm{c}$ & $4538.00 \mathrm{ab}$ & $1100.00 \mathrm{bc}$ & $213.20 \mathrm{~b}$ & $595.40 \mathrm{~b}$ \\
\hline & 100 & $2533.80 \mathrm{~b}$ & $3718.60 \mathrm{ab}$ & $1011.60 \mathrm{bc}$ & $1891.00 \mathrm{~b}$ & $3086.80 \mathrm{ab}$ & $1606.40 \mathrm{a}$ & $3506.00 \mathrm{ab}$ \\
\hline & 250 & $2723.60 \mathrm{~b}$ & $3989.80 \mathrm{ab}$ & $711.40 \mathrm{c}$ & $3337.20 \mathrm{ab}$ & $5682.40 \mathrm{~b}$ & $502.80 \mathrm{a}$ & $2743.60 \mathrm{ab}$ \\
\hline & 500 & $1485.40 \mathrm{~b}$ & $12418.40 \mathrm{~b}$ & $2100.60 \mathrm{~b}$ & $3147.00 \mathrm{ab}$ & $1021.00 \mathrm{c}$ & $652.40 \mathrm{a}$ & $14470.40 \mathrm{a}$ \\
\hline & 1000 & $7111.20 \mathrm{a}$ & $28241.60 \mathrm{ab}$ & $6695.40 \mathrm{a}$ & $8484.20 \mathrm{a}$ & $22307.53 \mathrm{a}$ & $1500.80 \mathrm{a}$ & $13961.20 \mathrm{a}$ \\
\hline & 20000 & $7293.00 \mathrm{a}$ & $13417.00 \mathrm{a}$ & $5637.60 \mathrm{a}$ & $5518.40 \mathrm{a}$ & $5450.60 \mathrm{ab}$ & $1844.40 \mathrm{a}$ & $4450.40 \mathrm{a}$ \\
\hline & C.V & 9.85 & 12.09 & 12.76 & 11.89 & 13.97 & 16.16 & 14.62 \\
\hline \multirow{7}{*}{$\mathrm{J} 2$} & 10 & 100 & $100 \mathrm{~b}$ & $52.00 \mathrm{~b}$ & 76 & 70 & $36.00 \mathrm{~b}$ & $144.00 \mathrm{~b}$ \\
\hline & 100 & 150 & $180 \mathrm{~b}$ & $96.00 \mathrm{ab}$ & 96 & 160 & $64.00 \mathrm{a}$ & $150.00 \mathrm{~b}$ \\
\hline & 250 & 240 & $70 \mathrm{~b}$ & $52.00 \mathrm{~b}$ & 90 & 220 & $120.00 \mathrm{a}$ & $144.00 \mathrm{~b}$ \\
\hline & 500 & 180 & $164 \mathrm{~b}$ & $144.00 \mathrm{ab}$ & 160 & 200 & $140.00 \mathrm{a}$ & $122.00 \mathrm{~b}$ \\
\hline & 1000 & 360 & $200 \mathrm{~b}$ & $260.00 \mathrm{a}$ & 300 & 280 & $160.00 \mathrm{a}$ & $460.00 \mathrm{a}$ \\
\hline & 20000 & 220 & $720 \mathrm{a}$ & $360.00 \mathrm{a}$ & 220 & 260 & $240.00 \mathrm{a}$ & $880.00 \mathrm{a}$ \\
\hline & C.V & 13.75 & 8.29 & 13.75 & 13.75 & 13.74 & 13.59 & 7.94 \\
\hline \multirow{7}{*}{$\begin{array}{l}\text { Población } \\
\text { Final }\end{array}$} & 10 & $40220.0 \mathrm{bc}$ & $7333.40 \mathrm{~b}$ & $5492.00 \mathrm{bc}$ & $77009.00 \mathrm{a}$ & $14080.00 \mathrm{bc}$ & 6276 & $13684.00 \mathrm{c}$ \\
\hline & 100 & $32897.6 \mathrm{ab}$ & $35680.00 \mathrm{a}$ & $20345.80 \mathrm{~b}$ & $18000.80 \mathrm{~b}$ & $36232.00 \mathrm{ab}$ & 19564 & $50218.20 \mathrm{ab}$ \\
\hline & 250 & $45551.0 \mathrm{ab}$ & $37284.00 \mathrm{a}$ & $10135.40 \mathrm{c}$ & $55891.60 \mathrm{a}$ & $58636.60 \mathrm{a}$ & 11123 & $41265.20 \mathrm{~b}$ \\
\hline & 500 & $19763.2 \mathrm{c}$ & $91068.80 \mathrm{a}$ & $26572.60 \mathrm{~b}$ & $32693.40 \mathrm{a}$ & $13871.40 \mathrm{c}$ & 10440 & $\begin{array}{c}151688.60 \\
a b\end{array}$ \\
\hline & 1000 & $81793.4 \mathrm{a}$ & $197700.00 \mathrm{a}$ & $84593.40 \mathrm{a}$ & $94352.40 \mathrm{a}$ & $290030.00 \mathrm{a}$ & 27560 & $238093.20 \mathrm{a}$ \\
\hline & 20000 & $116024.8 \mathrm{a}$ & $139120.00 \mathrm{a}$ & $77276.80 \mathrm{a}$ & $110186.60 \mathrm{a}$ & $72093.20 \mathrm{a}$ & 32490 & $98227.60 \mathrm{ab}$ \\
\hline & C.V & 7.88 & 6.1 & 7.88 & 7.27 & 11.83 & 9.37 & 7.17 \\
\hline \multirow{7}{*}{$\begin{array}{c}\text { RelacionPf/ } \\
\text { Pi }\end{array}$} & 10 & $402.2 \mathrm{a}$ & $73.334 \mathrm{a}$ & $54.92 \mathrm{a}$ & $770.09 \mathrm{a}$ & $140.8 \mathrm{a}$ & $62.76 \mathrm{a}$ & $136.84 \mathrm{a}$ \\
\hline & 100 & $32.898 \mathrm{~b}$ & $35.68 \mathrm{~b}$ & $20.346 \mathrm{~b}$ & $18.001 \mathrm{bc}$ & $36.232 \mathrm{ab}$ & $19.564 \mathrm{~b}$ & $50.218 \mathrm{a}$ \\
\hline & 250 & $18.22 \mathrm{c}$ & $14.914 \mathrm{c}$ & $4.054 \mathrm{~d}$ & $22.357 \mathrm{~b}$ & $23.455 \mathrm{bc}$ & $4.449 \mathrm{c}$ & $16.506 \mathrm{bc}$ \\
\hline & 500 & $3.953 \mathrm{c}$ & $18.214 \mathrm{c}$ & $5.315 \mathrm{bcd}$ & $6.539 \mathrm{c}$ & $2.774 \mathrm{~d}$ & $2.088 \mathrm{~cd}$ & $30.338 \mathrm{ab}$ \\
\hline & 1000 & $8.179 \mathrm{c}$ & $19.77 \mathrm{c}$ & 8.459 bc & $9.435 \mathrm{c}$ & $29.003 \mathrm{bc}$ & $2.756 \mathrm{~cd}$ & $23.809 \mathrm{bc}$ \\
\hline & 20000 & $5.801 \mathrm{c}$ & $6.956 \mathrm{c}$ & $3.864 \mathrm{~cd}$ & $5.509 \mathrm{bc}$ & $3.605 \mathrm{dc}$ & $1.625 \mathrm{~d}$ & $4.911 \mathrm{c}$ \\
\hline & C.V & 15.88 & 12.55 & 20.41 & 14.64 & 21.48 & 23.55 & 13.66 \\
\hline
\end{tabular}




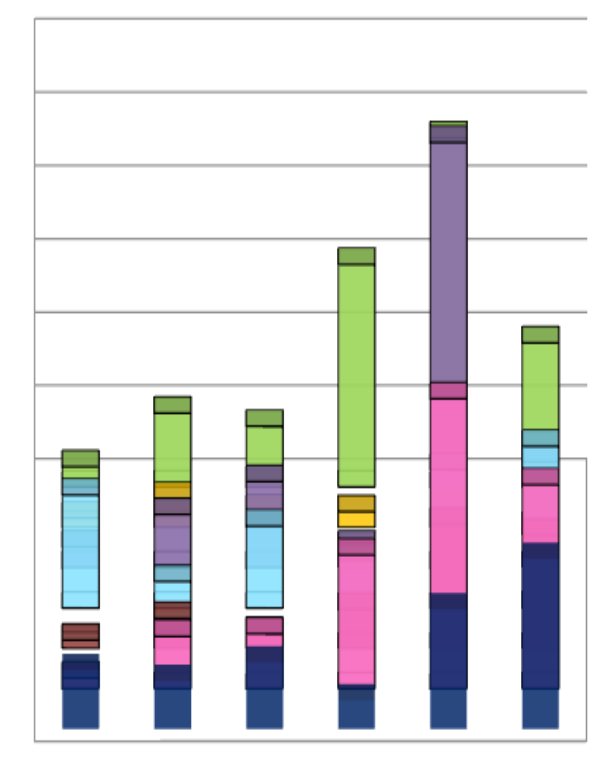

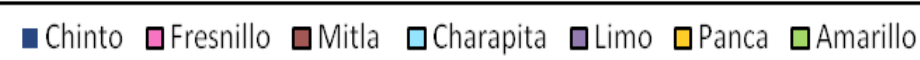

Figura 4. Población final en cultivares de Capsicum después de inoculados con diferentes densidades poblacionales de $M$. incognita.

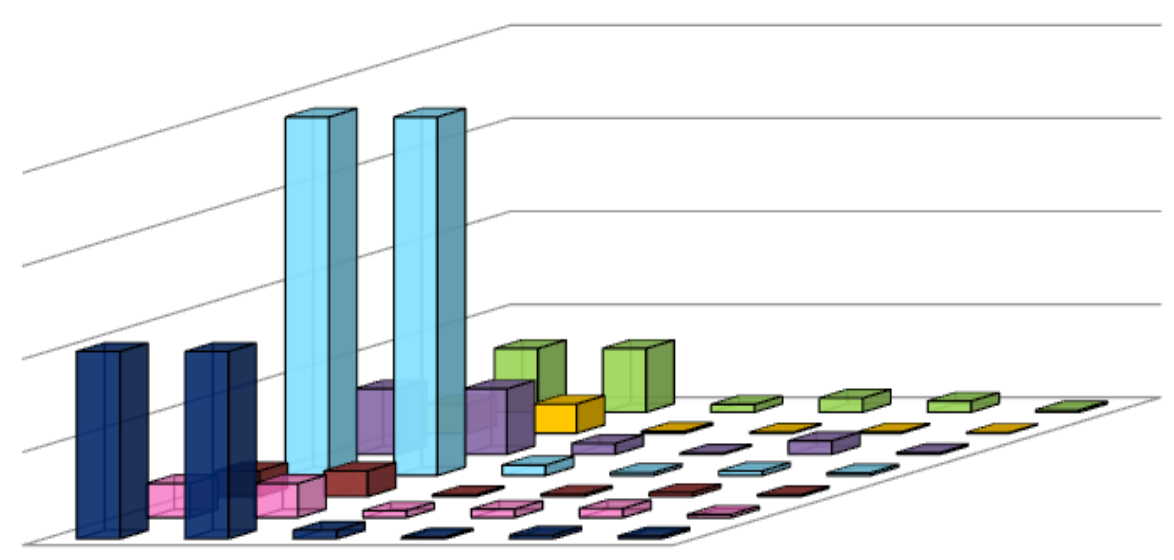

$\square$ Chinto $\square$ Fresnillo $\square$ Mitla $\square$ Charapita $\square$ Limo $\square$ Panca $\square$ Amarillo

Figura 5. Relación Pf/Pi en cultivares de Capsicum inoculados con diferentes densidades poblacionales de Meloidogyne incognita en cultivares de Capsicum. 
Según la escala de Evaluación de Taylor y Sasser, todos los cultivares estudiados son susceptibles a M. incognita. Se debe tener en cuenta que el sistema de evaluación, toma en cuenta la tasa de reproducción del nematodo en el sistema radicular de la planta y el grado de nodulación, sin embargo la escala del Proyecto Internacional Meloidogyne, utilizada con fines prácticos, en algunos casos, puede dar la impresión de un daño mayor cuando realmente no lo hay. Por esta razón resulta más conveniente el uso de la escala de Evaluación propuesta por V. Dropkin y modificada por Canto - Sáenz, donde se toma en cuenta el efecto del daño ocasionado por el nematodo y la tasa de reproducción del nematodo en la planta hospedante.

El comportamiento de la resistencia de los Capsicum estudiados, es similar al observado en diversos cultivares de tomate, donde pudo ser observada la variabilidad inter - e intraespecífica tanto en los cultivares resistentes como en susceptibles (IBPGR, 1983). El grado de resistencia que expresa el tomate frente al nematodo puede variar entre cultivares en función del genotipo del tomate receptor del gen $M i$, de la frecuencia de segregantes genéticos para el carácter de resistencia y la constitución alélica del carácter, por lo que, es posible obtener cultivares que expresen rangos intermedios de resistencia (8).

En el estudio realizado, los resultados obtenidos de peso seco del follaje y el peso fresco de raíces, fueron parámetros constantes utilizados para evaluar el comportamiento de los cultivares de Capsicum frente al ataque de $M$. incognita.

\section{Capsicum annum:}

Entre los cultivares de la especie Capsicum annum, el Mitla fue aquel en presentar mayor tolerancia a $M$. incognita. No se observaron diferencias estadísticas en los parámetros indirectos relacionados con el crecimiento y desarrollo de la planta, sin embargo observo una reducción del peso fresco conforme se incremento la densidad poblacional. En los parámetros directos, se observaron diferencias altamente significativas en el número de huevos por raíz, población y tasa de reproducción, siendo el cultivar Mitla un hospedante eficiente para el nematodo. El índice de nodulación promedio de acuerdo al Proyecto Internacional Meloidogyne fue igual a 4.33.

El cultivar Fresnillo presenta una tolerancia intermedia entre los tres cultivares de C. annuum estudiados. Los parámetros directos relacionados con la reproducción del nematodo en la planta presentaron diferencias altamente significativas. En este cultivar se observó una tendencia a incrementar el número de huevos por gramo de raíz entre las poblaciones inoculadas de 100 a 1000 huevos/ $100 \mathrm{cc}$. de suelo, mostrando una relación directa entre el número de huevos por gramo de raíz y la cantidad de nematodos inoculados. El índice de nodulación promedio para el cultivar Fresnillo fue igual a 4.67.
El cultivar Chinto, tiene la menor tolerancia a $M$. incognita; todos los parámetros relacionados con la reproducción del nematodo presentaron diferencias altamente significativas entre el testigo inoculado con 10 individuos/100 cc.de suelo y las otras densidades poblacionales; este cultivar presenta el mayor número de huevos por gramo de raíz comparado con los otros dos cultivares, teniendo diferencias altamente significativas. El número de individuos presentes en el sistema radicular es uno de los más altos observados junto con el cultivar Charapita (C. chinense). El índice de nodulación promedio, según el Proyecto Internacional Meloidogyne, es igual a 4.90 .

\section{Capsicum chinense}

Dentro de la especie $C$. chinense, el cultivar Panca es que el que presento mayor tolerancia a $M$. incognita, ningún parámetro relacionados con el crecimiento de la planta es afectado significativamente por las densidades poblacionales inoculadas. El cultivar Panca presenta el menor número de huevos por gramo de raíz entre los cultivares de la misma especie estudiados, encontrándose diferencias altamente significativas. Este cultivar presenta la menor tasa de reproducción (Pf/Pi) observada en todos los cultivares inoculadosa la mayor densidad poblacional inoculada de 2000 huevos por 100 cc.de suelo. El índice de nodulación promedio según el Proyecto Internacional Meloidogyne es igual a 4.73, siendo el menor índice de nodulación observado a la densidad de 10 huevos/100 cc.de suelo $(\mathrm{IN}=4)$.

El cultivar Limo presenta una resistencia moderada a $M$. incognita, el único parámetro del crecimiento de la planta que es afectado de forma significativa es el peso fresco del follaje, pero por su variabilidad no es considerado para determinar la reacción del cultivar ante la presencia del M. incognita.

Respecto al número de huevos por gramo de raíz, el cultivar Limo presenta diferencias altamente significativas entre el testigo inoculado y la densidad inoculada de 1000 huevos/cc.desuelo, donde se encontró como población final 69,386 huevos por gramo de raíz, siendo este valor el más alto registrado para este cultivar y el mayor valor observado a las diferentes densidades poblacionales inoculadas en los otros cultivares evaluados. La población final de nematodos en el cultivar Limo también presenta diferencias altamente significativas; estando a la densidad de 1000 huevos/100 cc.de suelo el mayor valor observado para este cultivar y para los cultivares pertenecientes a las otras especies de Capsicum en el estudio. El promedio de nodulaciónentre las diferentes densidades de inoculación de acuerdo al Proyecto Internacional Meloidogyne fue igual a 4.90.

Entre los cultivares de $C$. chinenseel Charapita es suceptible a $M$. incognita, el peso fresco de raíz es el único parámetro de crecimiento y desarrollo de planta en presentar diferencias significativas entre el testigo 
sin inocular y las densidades poblacionales inoculadas de 100,500 y 1000 huevos/100 cc de suelo. En el número de huevos por gramo de raíz, no fueron observadas diferencias significativas entre el testigo inoculado y las otras densidades poblacionales, las diferencias significativas encontradas en el ANVA, se encuentran entre la densidad poblacional de 100 huevos/100 cc de suelo y las densidades poblacionales inoculadas de 1000 y 2000 huevos/100 cc de suelo.

La cantidad de huevos por gramo de raíz y el numero de nematodos encontrados en el testigo inoculado fue el valor más alto registrado de todos los cultivares, lo que también fue reflejado en la tasa de reproducción, que fue la mayor registrada si se compara con los otros cultivares a las diferentes densidades poblacionales inoculadas; siendo el cultivar Charapita el que permite la mayor reproducción a bajas densidades poblacionales de $\mathrm{M}$. incognita. El índice de nodulación promedio según el Proyecto Internacional Meloidogyne fue 4.96.

\section{Capsicum baccatum cv. pendullum}

El cultivar Amarillo o Escabeche, no presentó diferencias significativas en los parámetros relacionados con el crecimiento de planta. En el número de huevos por gramos de raíz y la población final, se observan diferencias altamente significativas entre el testigo inoculado y las otras densidades poblacionales. La tasa de reproducción (Pf/Pi), al igual que en todos los cultivares estudiados,es mayor a 1.5 lo que hace a este cultivar un hospedante eficiente para M. incognita.

\section{Conclusiones}

Todos los cultivares que participaron en el estudio, se comportaron como hospedantes eficientes para Meloidogyne incognita en todos los niveles poblacionales inoculados para este ensayo.

Los cultivares Mitla, Fresnillo (C. annuum) y Panca ( $C$. chinense) presentaron mayor tolerancia al ataque del nematodo, Meloidogyne incognita. Los cultivares Chinto y Limo presentaron una tolerancia moderada al daño producido por este nematodo para las condiciones del ensayo.

Los cultivares Charapita y Amarillo se comportaron como susceptibles a altas densidades poblacionales de $M$. incognita, para este ensayo.

Ninguno de los cultivares estudiados presento resistencia o hipersuceptibilidad a $M$. incognitapara este ensayo.

La escala de evaluación propuesta por V. Dropkin y modificada por Canto - Sáenz, es la más eficiente para determinar la reacción de la planta ante el daño ocasionado por Meloidogyne incognita,

La escala visual de Zeck es útil para determinar el grado de nodulación en las raíces, en las condiciones en las que fue realizado el estudio.
Bajo las condiciones en las que se realizó el estudio, todos los cultivares evaluados presentan diferentes niveles de tolerancia Meloidogyne incognita.

\section{Recomendaciones}

Evaluar los cultivares en condiciones de campo, para determinar su comportamiento ante las poblaciones de Meloidogyne incognita.

Evaluar el comportamiento de los cultivares ante diferentes aislamientos y razas de Meloidogyne incognita.

Determinar el tipo de resistencia que presentan los cultivares (poligénica, monogénica) y los genes involucrados.

Evitar el uso en monocultivo de un cultivar resistente y/o tolerante por que puede ocasionar una selección de poblaciones de nematodos que quiebren la resistencia, volviéndolas susceptibles con el tiempo susceptibles.

\section{Literatura citada.}

Barker, K. R. 1985. Nematode Extractions and Bioassays. In An Advance Treatiise on Meloidogyne. Vol II: Methodology. North Carolina State University Graphics: Raleigh, North Carolina, USA.

Boerma, H. R. and R. S. Hussey. 1992. Breeding Plants for Resistance of Nematodes. J. Nematol. 24: 242 252.

Bosland, P. W. 1996. Capsicums: Innovative uses of ancient crop. En Janick J. (ed). Progress in new crops. ASHS Press, Arlington, V. A. p. 479-487.

Casseres E. 1966. Producción de Hortalizas. Primera Edición. Instituto Interamericano de Ciencias Agrícolas de la OEA. Lima. Perú.

Canto Saenz M. 1985. Nature of Resistance to Meloidogyne incognita (Kofoid \& White, 1919) Chitwood, 1949. In An Advance Treatiise on Meloidogyne Vol. I: Biology and Control. North Carolina State University Graphics: Raleigh, North Carolina, USA.

De Souza-Sobrinho F., Maluf W., Gomes L. A. A. and Campos P. L. 2000. Inheritance of resistance to Meloidogyne incognita race 2 in the hot pepper cultivar Carolina Cayenne (Capsicum annuum L.). Departamentos de Biologia, Agricultura, and Fitopatologia, Universidade Federal de Lavras. Brazil.

Di Vito, M. 1986. Population densities of Meloidogyne incognita and growth of susceptible and resistant pepper plants. Nematol. Mediterr. 14:217-221.

Di Vito, M., Saccardo,F., Errico, A., Zema, V., and Zaccheo, G. 1993. Genetics of resistance to root-knot nematodes (Meloidogyne spp.) in Capsicum chacoense, C. chinense, and C. frutescens. J. Genet. Breed. 47:23-26. 
Espino, L. A. 2002. Reacción de quince cultivares de tomate Lycopersicon esculentum Mill a Meloidogyne incognita (Kofoid \& White) Chitwood y a Fusarium oxysporum Schelechtend: Fr. F. sp. lycopersici (Sacc) W. C. Zinder \& H. N. Hans en invernadero.

Fassuliotis, G.1979. Plant breeding for root-knot nematode resistance, p: 425-453. In: Rootknot Nematodes (Meloydogine sp.) Systematics, biology, and control. Eds. F. Lamberti and C.E. Taylor. Academic Press, New York.

Hartman, K. M. and J. N. Sasser. 1985. Identification of Meloidogyne species on the basis of differential host test and perineal - pattern morphology. In An Advance Treatiise on Meloidogyne. Vol II: Methodology. North Carolina State University Graphics: Raleigh, North Carolina, USA.

Heiser, Ch. and P. G. Smith. 1953. The Cultivated Capsicums peppers. Bot., Econ. 7: $214-227$.

Hussey, R. and K. R. Barker. 1973. A Comparasion of Methods of Collecting inoculo of Meloidogyne spp. Including a New Technique. Plant. Dis. Rep.57:1025 1028.

IBPGR. 1983. Genetics Resources of Capsicum - A global Plan Action. International Board for Plant Genetic Resources AGPG / IBPGR /82 / 12. Rome. Italy. 49 p.

Jensen H. J. 1972. Nematode Pest of Vegetable and Related Crops 377 - 408 pp. In J. M. Webster [ed]. Economic Nematology. Academic Press. London.

Jones, J. B., Jones, J. P., Stall, R. E., and Zitter, T. A. 1991. Compendium of Tomato Diseases. The American Phytopathological Society. APS Press. St. Paul, MN. $73 \mathrm{pp}$.

Noe, J. P. 1985. Analysis and Interpretation of data from Nematological Experiments. In An Advance Treatise on Meloidogyne. Vol II: Methodology. North Carolina State University Graphics: Raleigh, North Carolina, USA.

Orbegozo, A. G. 1954. Reseña del Cultivo, Identificación, Botánica y Comparativo del Rendimiento en fresco de variedades de ají. Tesis Ingeniero Agrónomo. Escuela Nacional de Agricultura "La Molina". Lima. Perú.

Sasser J. N. and D. W. Freckman. 1987. A world perspective on nematology: the role of the society. In: J.A. Veech and D.W. Dickson, Editors, Vistas on Nematology, Society of Nematologist, Hyattsville MD. pp. 7-14.

Taylor, A. and J. N. Sasser. 1978. Biology, Identification and Control of root - knot Nematodes (Meloidogyne spices). North Carolina State University Graphics, USA.

Zeck, W. M. 1971. A rating scheme for field Evaluation root - know nematode infestations. Pflanzenschutz Nachrichten. 24: $141-144$. 